\title{
Enhancement in Dissolution Rate of Piroxicam by Two Micronization Techniques
}

\author{
J. Varshosaz*, A. Khajavinia, M. Ghasemlu, \\ E. Ataei, K. Golshiri, and I. Khayam \\ Department of Pharmaceutics, Faculty of Pharmacy and Novel Drug Delivery Systems \\ Research Center, Isfahan University of Medical Sciences, Isfahan, P.O. Box 81745-359, Iran
}

\begin{abstract}
Piroxicam is a nonsteroidal anti-inflammatory drug that is practically insoluble in water. The oral absorption rate of piroxicam is dependent on its dissolution rate in the Gl tract. The aim of this study was to enhance the dissolution of piroxicam by a microcrystallization technique. The preparation of microcrystals of piroxicam was done by two methods, solvent change and $\mathrm{pH}$ shift. In the solvent-change method, the drug was dissolved in acetone, and the stabilizer was dissolved in water. The aqueous phase was added to acetone under homogenization in an ice bath for $1 \mathrm{~min}$. In the pH-shift method, the drug and stabilizer were both dissolved in $0.1 \mathrm{~N} \mathrm{NaOH}$ ( $\mathrm{pH}$ 12) using homogenization. The $\mathrm{pH}$ was adjusted to 3 using $0.1 \mathrm{~N}$ hydrochloric acid. Dissolution testing was carried out in a hydrochloric acid medium using the rotating basket method. Particle size and morphology, FTIR, DSC, XRD, and surface area of the microcrystals were studied. The effects of drug and stabilizer concentration and homogenization rate on particle size and dissolution efficiency were studied statistically using a D-optimal design. The dissolution efficiency in both methods was increased about 3- to 4-fold. The particle size in both methods was decreased in comparison with untreated drug. Maximum dissolution and minimum particle size were obtained by the solvent-change method. According to the results, both microcrystallization methods are effective in the modification of the crystalline the habit of piroxicam.
\end{abstract}

\section{INTRODUCTION}

P oor solubility is one of the major challenges in drug development today (1). An estimated $40 \%$ of drugs fall under BCS Class 2 (low solubility and high permeability) or Class 4 (low solubility and low permeability) $(2,3)$. These drugs show limited bioavailability because of their low solubility (4). Piroxicam, one of the most potent nonsteroidal anti-inflammatory drugs, is a BCS Class 2 drug with high membrane permeability but low water solubility (5). Different techniques have been studied to enhance the dissolution rate of piroxicam. Cavallaria et al. (6) used beta-cyclodextrin to improve the dissolution of piroxicam powder by wet granulation. Results showed advantages such as better powder flow and compressibility and improved bioavailability. Other methods such as liquid-solid compacts $(5,7)$, wet granulation using betalactose and PVP $(8)$, and solid dispersions $(9,10)$ have been proposed to enhance the dissolution rate of piroxicam.

Micronization is an effective way to enhance the dissolution rate of poorly water-soluble drugs $(11,12)$. However, mechanical methods such as milling or grinding have inevitable drawbacks, including the tendency of the produced particles to agglomerate, reducing the available surface area (13). In addition, the potential for physical instability or chemical degradation from disruptions in the crystal lattice $(14,15)$ and the high energy input makes this micronization method extremely inefficient (16). Several other methods have been introduced to produce

*Corresponding author. micron-sized drug particles such as high-pressure homogenization (17), supercritical fluid micronization $(18,19)$, liquid-solid techniques (20), and hydrosol formation (11).

In situ micronization has been proposed recently to overcome the above-mentioned problems of mechanical techniques. Since particles are prepared directly in the micronized state (21) and the newly formed microcrystal surface is simultaneously stabilized by a stabilizer (usually a polymer), particles have a much lower tendency for crystal growth and agglomeration (11).

Two methods of microcrystallization are $\mathrm{pH}$ shift and solvent change. In the solvent-change method, an organic solvent and an aqueous solvent with a stabilizing agent are used. The drug microcrystals are formed by mixing the aqueous solution of the stabilizer with the organic drug solution; this leads to a reduction of interfacial tension, since the stabilizing agent would be adsorbed on the precipitated drug particles. A drug powder with a high drug load is obtained after drying this dispersion (22). Disodium cromoglycate microcrystals have been prepared for pulmonary delivery using this method (22). Zimmermann et al. (23) reported that the adsorption of pharmaceutical excipients onto microcrystals of siramesine hydrochloride changes the physicochemical properties of the drug such as particle size, morphology, and dissolution rate. Aspirin, mebutamate, and quinine sulfate microcrystals with a particle size of less than $10 \mu \mathrm{m}$ were prepared using the solvent-change method (24).

In the pH-shift method, an aqueous solution is used instead of organic solvent. A supersaturated solution is 
Table 1. Different Process Variables and Respective Levels in Micronization Technique (pH-Shift and Solvent-Change Methods)

\begin{tabular}{|c|c|c|c|}
\hline \multirow[b]{2}{*}{ Micronization Technique } & \multirow[b]{2}{*}{ Variables } & \multicolumn{2}{|c|}{ Levels } \\
\hline & & Lower Limit & Upper Limit \\
\hline \multirow{3}{*}{$\mathrm{pH}$ shift } & A: Drug concentration (\%) & 0.3 & 0.6 \\
\hline & B: Stabilizer concentration (\%) & 0.03 & 0.06 \\
\hline & C: Homogenization speed (rpm) & 17500 & 21500 \\
\hline \multirow{4}{*}{ Solvent change } & A: Drug concentration (\%) & 0.3 & 0.6 \\
\hline & B: Stabilizer concentration (\%) & 0.03 & 0.06 \\
\hline & C: Homogenization speed (rpm) & 17500 & 21500 \\
\hline & D: Stabilizer type & Brij 35 & Poloxamer 188 \\
\hline
\end{tabular}

prepared to precipitate the drug microcrystals using $\mathrm{pH}$ dependent solubility. Kim et al. (16) reported an increased dissolution rate and uniform size distribution for indomethacin, which has a pH-dependent solubility.

In the present study, two techniques of solvent change and $\mathrm{pH}$ shift are compared for size reduction, and hence the enhancement of dissolution and bioavailability of piroxicam.

\section{MATERIALS AND METHODS Materials}

Piroxicam was supplied from Darupakhsh Company (Iran). Brij35 (Fluka, USA), Poloxamer 188 or Pluronic F68 (Sigma, USA), acetone, ethanol, $\mathrm{HCl}$, and $\mathrm{NaOH}$ were all from Merck Chemical Company (Germany). All chemicals were of analytical grade.

\section{In Situ Micronization Technique}

After different process variables including the concentration of the drug and stabilizer, homogenization speed, and stabilizer type were determined, two levels were established for each variable. By changing three process variables for the $\mathrm{pH}$-change method and four variables for the solvent-change method (Table 1), each at two levels, eighteen and twenty-four different formulations were designed by a D-optimal design, respectively, using Design Expert software (Version 7.2, US).

Preparation of Piroxicam Microcrystals Using pH-Shift Method

Piroxicam microcrystals were prepared by reducing the $\mathrm{pH}$ level of the piroxicam solution to form a micron-size dispersion of the drug due to low solubility of piroxicam in lower $\mathrm{pH}$ values.

The drug was dissolved in $100 \mathrm{~mL}$ of $0.1 \mathrm{~N} \mathrm{NaOH}(\mathrm{pH}$ 12) containing Brij35 as the stabilizer. After the drug was completely dissolved using an ultra-homogenizer (Ultra Turrax-T25 Basic IKA, Germany), the $\mathrm{pH}$ of the solution was reduced from 12 to 3 within one minute by the addition of $0.1 \mathrm{~N} \mathrm{HCl}$ in an ice bath. The lower $\mathrm{pH}(\mathrm{pH} 3)$ was selected based on preliminary studies in which reducing the $\mathrm{pH}$ of the dispersion to lower values had not changed the amount of precipitate. After the spontaneous formation of the micron-size dispersion of the drug, the mixture was transferred to a rotary evaporator (Buchi, Switzerland) and stirred for one hour at $40^{\circ} \mathrm{C}$. After that, the aqueous suspension was frozen at $-70^{\circ} \mathrm{C}$ for $24 \mathrm{~h}$ and freeze-dried (Christ Alpha 4.2 LD, Germany) at 0.001 bar for $24 \mathrm{~h}$.

Eighteen different formulations described in Table 2 were prepared by this procedure.

\section{Preparation of Piroxicam Microcrystals Using Solvent-} Change Method

The process was carried out by instantaneously mixing two liquids in the presence of Brij35 or poloxamer188 as stabilizing agents. The process was performed in an ice bath. In the first step, the drug was dissolved in $100 \mathrm{~mL}$ of acetone (as the solvent) and the stabilizing agent in 100 $\mathrm{mL}$ of water (as nonsolvent). The nonsolvent was poured rapidly into the drug solution under stirring at different homogenization speeds ranging from 17500 to 21500 rpm using an ultra-homogenizer. A micron size, fine dispersion formed spontaneously. The dispersion was dried using the previous method. Twenty-four different formulations were prepared using this procedure (Table 3 ).

\section{Scanning Electron Microscopy (SEM)}

Electron micrographs of crystals were obtained using a scanning electron microscope (PHILIPS X L30, Netherlands). The specimens were mounted on a metal stub with double- sided adhesive tape and coated under vacuum with gold in an argon atmosphere prior to observation.

\section{Particle Size Analysis}

A laser diffraction particle size analyzer (Malvern, long bed ver. 2.15, UK) was used to measure the size distribution and the mean particle size diameter of the prepared microcrystals and the pure drug powder by dispersing the particles in double-distilled water.

\section{X-ray Powder Diffraction}

The sample holder of the X-ray diffractometer (Bruker, D8 ADVANCE, Germany) was filled with the ground 
Table 2. Particle Size and $D E_{150}$ of Piroxicam Microcrystals Prepared by Solvent-Change Method with a D-Optimal Design

\begin{tabular}{|c|c|c|}
\hline Formulation Code & Particle size \pm SD ( $\mu \mathrm{m})$ & $\mathrm{DE}_{150} \pm \mathrm{SD}(\%)$ \\
\hline Untreated piroxicam & $15.6 \pm 37.4$ & $24.0 \pm 4.4$ \\
\hline$P_{0.52} B_{0.05} S_{19500}$ & $3.5 \pm 0.1$ & $52.1 \pm 6.5$ \\
\hline $\mathrm{P}_{0.6} \mathrm{P}_{0.06} \mathrm{~S}_{19500}$ & $1.1 \pm 0.1$ & $65.3 \pm 5.5$ \\
\hline$P_{0.3} B_{0.06} S_{17500}$ & $0.9 \pm 0.2$ & $65.0 \pm 4.0$ \\
\hline $\mathrm{P}_{0.3} \mathrm{~B}_{0.06} \mathrm{~S}_{19500}$ & $3.9 \pm 0.3$ & $70.2 \pm 4.2$ \\
\hline $\mathrm{P}_{0.45} \mathrm{~B}_{0.03} \mathrm{~S}_{21500}$ & $0.4 \pm 0.0$ & $69.3 \pm 5.9$ \\
\hline $\mathrm{P}_{0.6} \mathrm{P}_{0.03} \mathrm{~S}_{21500}$ & $14.5 \pm 0.4$ & $64.0 \pm 1.8$ \\
\hline $\mathrm{P}_{0.6} \mathrm{P}_{0.03} \mathrm{~S}_{17500}$ & $3.9 \pm 1.6$ & $51.4 \pm 3.1$ \\
\hline $\mathrm{P}_{0.3} \mathrm{P}_{0.06} \mathrm{~S}_{17500}$ & $3.3 \pm 0.0$ & $56.1 \pm 3.3$ \\
\hline $\mathrm{P}_{0.6} \mathrm{~B}_{0.06} \mathrm{~S}_{21500}$ & $0.7 \pm 0.1$ & $82.5 \pm 2.3$ \\
\hline $\mathrm{P}_{0.45} \mathrm{~B}_{0.03} \mathrm{~S}_{21500}$ & $1.4 \pm 0.1$ & $70.2 \pm 1.4$ \\
\hline $\mathrm{P}_{0.6} \mathrm{~B}_{0.06} \mathrm{~S}_{17500}$ & $14.2 \pm 0.1$ & $46.0 \pm 3.0$ \\
\hline $\mathrm{P}_{0.6} \mathrm{~B}_{0.03} \mathrm{~S}_{17500}$ & $2.3 \pm 0.4$ & $55.0 \pm 2.2$ \\
\hline $\mathrm{P}_{0.3} \mathrm{~B}_{0.03} \mathrm{~S}_{17500}$ & $1.8 \pm 0.4$ & $67.0 \pm 1.5$ \\
\hline $\mathrm{P}_{0.45} \mathrm{~B}_{0.04} \mathrm{~S}_{18500}$ & $12.3 \pm 0.3$ & $66.3 \pm 1.2$ \\
\hline $\mathrm{P}_{0.6} \mathrm{~B}_{0.03} \mathrm{~S}_{19500}$ & $9.3 \pm 0.1$ & $44.0 \pm 0.9$ \\
\hline $\mathrm{P}_{0.3} \mathrm{P}_{0.03} \mathrm{~S}_{17500}$ & $1.3 \pm 0.0$ & $71.6 \pm 2.3$ \\
\hline $\mathrm{P}_{0.3} \mathrm{P}_{0.06} \mathrm{~S}_{21500}$ & $3.3 \pm 0.3$ & $87.2 \pm 6.5$ \\
\hline $\mathrm{P}_{0.6} \mathrm{~B}_{0.06} \mathrm{~S}_{21500}$ & $0.3 \pm 0.0$ & $85.3 \pm 2.4$ \\
\hline $\mathrm{P}_{0.3} \mathrm{~B}_{0.04} \mathrm{~S}_{21500}$ & $1.4 \pm 0.4$ & $86.2 \pm 0.7$ \\
\hline $\mathrm{P}_{0.6} \mathrm{P}_{0.03} \mathrm{~S}_{21500}$ & $9.3 \pm 0.0$ & $78.0 \pm 1.6$ \\
\hline $\mathrm{P}_{0.3} \mathrm{P}_{0.03} \mathrm{~S}_{19500}$ & $14.1 \pm 0.2$ & $71.5 \pm 5.1$ \\
\hline $\mathrm{P}_{0.45} \mathrm{P}_{0.04} \mathrm{~S}_{19500}$ & $10.2 \pm 0.0$ & $69.4 \pm 1.3$ \\
\hline $\mathrm{P}_{0.3} \mathrm{~B}_{0.04} \mathrm{~S}_{21500}$ & $1.5 \pm 0.1$ & $84.1 \pm 0.7$ \\
\hline $\mathrm{P}_{0.3} \mathrm{P}_{0.06} \mathrm{~S}_{21500}$ & $1.1 \pm 0.2$ & $73.4 \pm 4.0$ \\
\hline
\end{tabular}

sample powder and then smoothed with a spatula. X-ray diffraction patterns of untreated drug, microcrystals of piroxicam, and physical mixtures of the drug and excipients were obtained over a range of 5-60 $2 \theta$ by exposing the samples to $\mathrm{CuK} \alpha$ radiation.

\section{Fourier Transform Infrared (FTIR) Spectroscopy}

Fourier transform infrared spectra were obtained by FTIR spectrophotometry (Rayleigh, WQF-S10, China) over a scanning range of $450-4000 \mathrm{~cm}^{-1}$. Samples were prepared by mixing with $\mathrm{KBr}$ powder and compressing into a disk by hydraulic pressure.

\section{Thermal Analysis}

Differential scanning calorimetric tests were performed to investigate potential incompatibilities between the drug and the stabilizer and to monitor the purity of piroxicam microcrystals. The DSC thermograms of the samples (3-6 mg) were recorded using a thermal
Table 3. Particle Size and $D E_{150}$ of Piroxicam Microcrystals Prepared by $\mathrm{pH}$-Shift Method with a D-Optimal Design

\begin{tabular}{|c|c|c|}
\hline Formulation Code & Particle size \pm SD $(\mu \mathrm{m})$ & $\mathrm{DE}_{150} \pm \mathrm{SD}(\%)$ \\
\hline $\mathrm{P}_{0.6} \mathrm{~B}_{0.06} \mathrm{~S}_{21500}$ & $3.2 \pm 0.1$ & $63.4 \pm 3.3$ \\
\hline$P_{0.6} B_{0.03} S_{21500}$ & $3.7 \pm 0.3$ & $48.5 \pm 0.9$ \\
\hline $\mathrm{P}_{0.3} \mathrm{~B}_{0.06} \mathrm{~S}_{19500}$ & $3.1 \pm 0.2$ & $52.0 \pm 1.2$ \\
\hline$P_{0.6} B_{0.03} S_{21500}$ & $6.1 \pm 0.2$ & $57.2 \pm 1.5$ \\
\hline $\mathrm{P}_{0.3} \mathrm{~B}_{0.03} \mathrm{~S}_{17500}$ & $2.7 \pm 0.2$ & $74.1 \pm 0.9$ \\
\hline$P_{0.6} B_{0.04} S_{19500}$ & $3.5 \pm 0.0$ & $73.3 \pm 2.3$ \\
\hline $\mathrm{P}_{0.6} \mathrm{~B}_{0.03} \mathrm{~S}_{17500}$ & $4.7 \pm 0.6$ & $71.4 \pm 1.5$ \\
\hline $\mathrm{P}_{0.6} \mathrm{~B}_{0.06} \mathrm{~S}_{17500}$ & $2.9 \pm 0.6$ & $68.1 \pm 3.6$ \\
\hline $\mathrm{P}_{0.3} \mathrm{~B}_{0.04} \mathrm{~S}_{21500}$ & $3.6 \pm 0.4$ & $68.2 \pm 4.1$ \\
\hline $\mathrm{P}_{0.45} \mathrm{~B}_{0.04} \mathrm{~S}_{17500}$ & $3.8 \pm 0.1$ & $59.5 \pm 2.0$ \\
\hline $\mathrm{P}_{0.3} \mathrm{~B}_{0.06} \mathrm{~S}_{21500}$ & $2.4 \pm 0.1$ & $73.3 \pm 2.1$ \\
\hline $\mathrm{P}_{0.3} \mathrm{~B}_{0.03} \mathrm{~S}_{21500}$ & $6.3 \pm 0.3$ & $63.2 \pm 2.1$ \\
\hline $\mathrm{P}_{0.45} \mathrm{~B}_{0.03} \mathrm{~S}_{19500}$ & $2.5 \pm 0.3$ & $74.0 \pm 5.7$ \\
\hline $\mathrm{P}_{0.3} \mathrm{~B}_{0.06} \mathrm{~S}_{17500}$ & $5.4 \pm 0.2$ & $78.3 \pm 2.2$ \\
\hline $\mathrm{P}_{0.6} \mathrm{~B}_{0.03} \mathrm{~S}_{17500}$ & $5.3 \pm 0.3$ & $70.0 \pm 2.1$ \\
\hline $\mathrm{P}_{0.45} \mathrm{~B}_{0.05} \mathrm{~S}_{20500}$ & $2.6 \pm 0.0$ & $74.4 \pm 1.6$ \\
\hline $\mathrm{P}_{0.3} \mathrm{~B}_{0.03} \mathrm{~S}_{17500}$ & $2.3 \pm 0.2$ & $73.2 \pm 1.2$ \\
\hline $\mathrm{P}_{0.6} \mathrm{~B}_{0.06} \mathrm{~S}_{17500}$ & $3.2 \pm 0.5$ & $68.2 \pm 3.3$ \\
\hline
\end{tabular}

analysis system (Mettler, TA400, Germany) calibrated with indium standard. The samples were heated at a scanning rate of $10^{\circ} \mathrm{C} / \mathrm{min}$ in an aluminum pan under a nitrogen atmosphere. A similar empty pan was used as the reference.

\section{Specific Surface Area}

The specific surface areas of the untreated drug and microcrystals were determined using the gas adsorption technique. Calculations were carried out according to the Brunauer-Emmett-Teller (BET) equation (25).

\section{Dissolution Studies}

The dissolution rate of $10 \mathrm{mg}$ of piroxicam crystals was determined using dissolution Apparatus 1 (basket) method (Erweka, Germany) at $37 \pm 0.5^{\circ} \mathrm{C}$ and stirring at $50 \mathrm{rpm}$. The dissolution medium was $900 \mathrm{~mL}$ of $0.1 \mathrm{~N} \mathrm{HCl}$. Samples were withdrawn from the dissolution vessels at selected time intervals $(5,10,15,20,30,45,60,90,120$, and $150 \mathrm{~min}$ ), and piroxicam was quantified at $328.8 \mathrm{~nm}$ with a UV spectrophotometer (Shimadzu, Japan). Each sample was replaced with fresh dissolution medium. Dissolution efficiency up to $150 \mathrm{~min}\left(D E_{150}\right)$ was calculated according to (26):

$$
D E \%=\frac{\int_{0}^{t} y d t}{y_{100} t} \times 100
$$



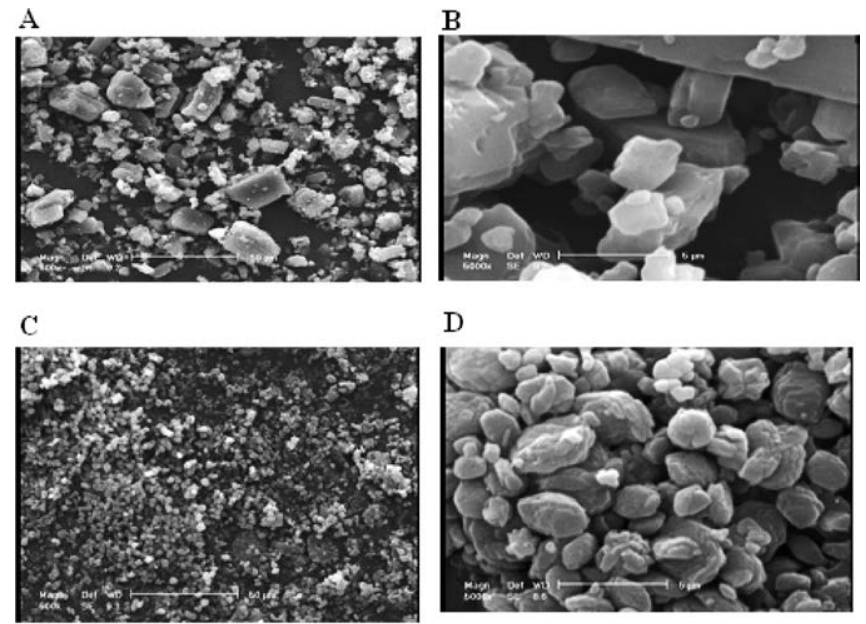

$\mathrm{E}$

$\mathrm{D}$

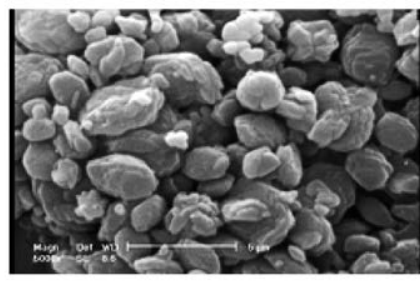

F
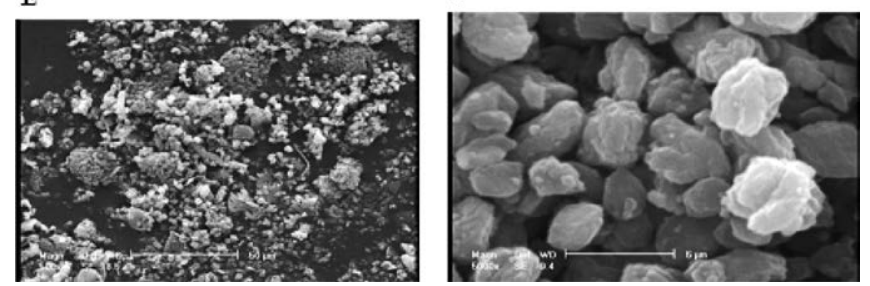

Figure 1. SEM micrographs of untreated piroxicam powder $(A) \times 500$ and (B) $\times 5000$; optimized microcrystals of piroxicam prepared by solventchange method $(C) \times 500$ and $(D) \times 5000$; and optimized microcrystals of piroxicam prepared by $\mathrm{pH}$-shift method $(E) \times 500$ and $(F) \times 5000$.

\section{RESULTS AND DISCUSSION}

\section{Microcrystallization of Piroxicam}

Three water-miscible solvents (i.e., acetone, methanol, and ethanol) were evaluated as potential solvents of piroxicam for the solvent-change method. Acetone was selected for the microcrystallization process because it can solubilize larger quantities of the drug.

Microcrystallization produces new surfaces and an increased energy of the system. A protective stabilizer prevents aggregation by steric hindrance between the particles. Different stabilizers such as HPMC, Brij35, Tween 80 , gelatin, chitosan, hydroxypropyl cellulose, poloxamer 188 , PEG 6000, and polyvinyl pyrrolidone have been studied as the protective polymers (27). Among these, $\mathrm{HPMC}$ and Brij35 at $0.05 \%$ and $0.1 \%$ concentrations yielded smaller particles with less aggregation (27). If the precipitation process is carried out without a stabilizer, the crystal growth would be greatly accelerated. This could be prevented by the adsorption of a stabilizer to the newly formed hydrophobic surface of the microcrystals. Thus polymers with more hydrophobic groups such as HPMC and methyl hydroxylethyl cellulose have a greater ability to stabilize the smaller particles than polymers with more hydrophilic properties such as HPC (17). This is due to better interaction and more similarity between the hydrophobic surface of the stabilizer and the particles. Poloxamer has the hydrophobic group of polyoxypropylene, and
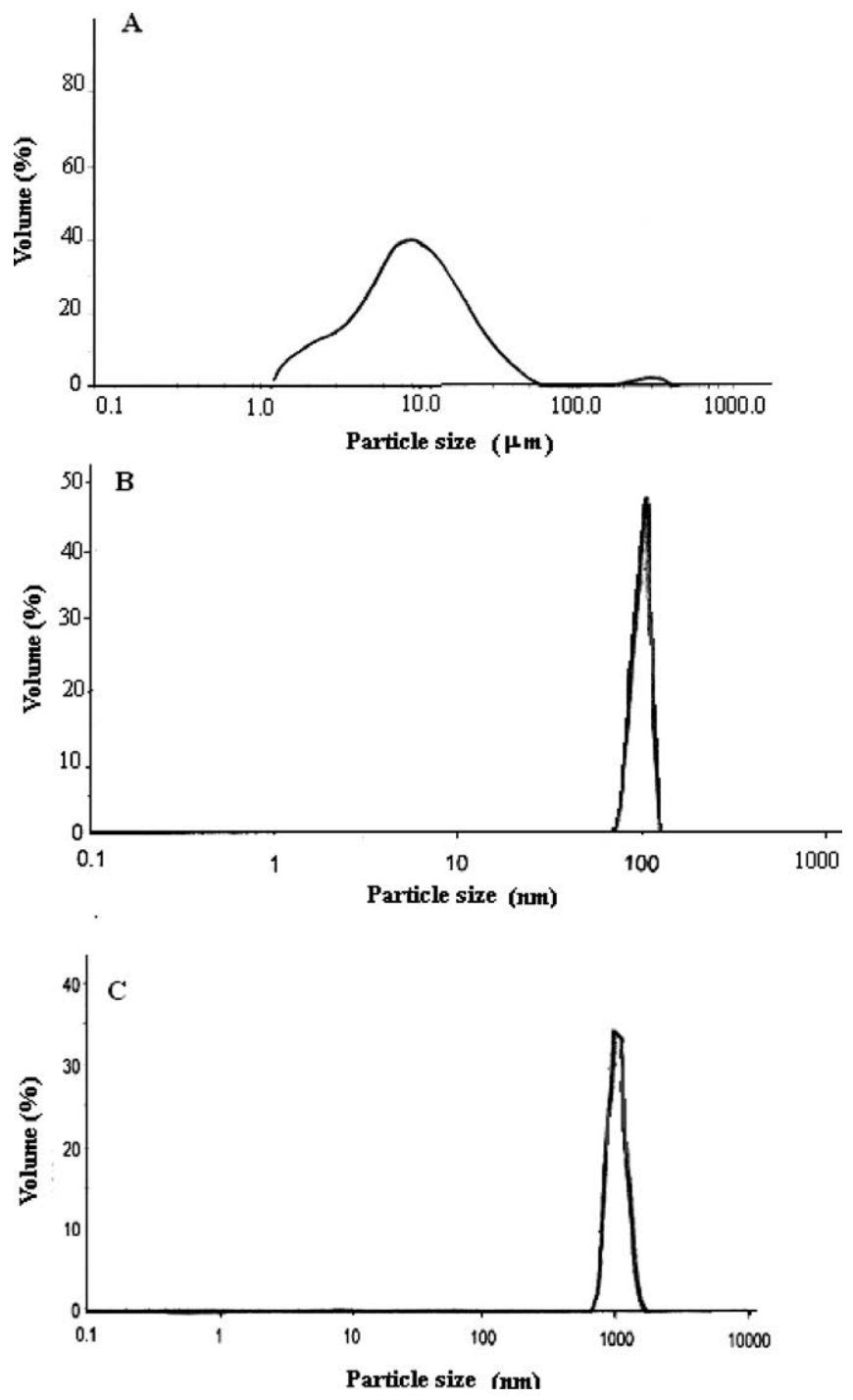

Figure 2. Particle size distribution of $(A)$ untreated piroxicam powder, (B) optimized microcrystals of piroxicam prepared by solvent-change method, and $(\mathrm{C})$ optimized microcrystals of piroxicam prepared by $\mathrm{pH}$-shift method.

Brij35 is a nonionic surfactant with a hydrophile-lipophile balance (HLB) of 16.9 (28), a suitable combination for stabilization of the microcrystals. In the solvent-change method, Brij35 produced smaller particles than poloxamer, and accordingly, it was the only studied stabilizer in the pH-shift method (Tables 2 and 3).

\section{SEM Morphology}

Micrographs of the untreated piroxicam powder and microcrystals of the optimum formulations of the two methods are shown in Figure 1. The untreated piroxicam particles are angular and cubic or rod-shaped, whereas the microcrystal particles have nearly spherical shapes and are obviously smaller. The nearly spherical microcrystals of piroxicam can result in better powder flow and fewer problems in producing solid dosage forms (27). In contrast, the pure piroxicam powder particles (Figure 1) 
are angular, rod-shaped cubic, and polymorphic, causing difficulties in powder flow.

\section{Particle Size Distribution of Microcrystals}

The size distribution of standard piroxicam powder and microcrystals of the optimum formulations of the two methods are shown in Figure 2. Tables 2 and 3 show the mean particle size of all formulations prepared by the two methods.

Untreated piroxicam powder had the largest particle size with a mean diameter of $15.66 \pm 37.36 \mathrm{~mm}$. As shown in Table 2, the smallest mean particle size in the solventchange method was obtained from the formulation $\mathrm{P}_{0.6} \mathrm{~B}_{0.06} \mathrm{~S}_{21500}$. However, the smallest mean particle size in the $\mathrm{pH}$-shift method was obtained from the formulation $\mathrm{P}_{0.3} \mathrm{~B}_{0.03} \mathrm{~S}_{17500}$.

Based on a quadratic model fitted by Design Expert software to particle size data, the following equations were obtained to predict the average particle sizes in terms of different process variables for solvent change $\left(\mathrm{Y}_{1}\right)$ and $\mathrm{pH}$ shift $\left(\mathrm{Y}_{2}\right)$ methods:

$$
\begin{aligned}
& Y_{1}=10.21+0.2 A-2.59 B+0.055 C+1.66 D+8.50 A^{2}+9.58 B^{2}- \\
& 2.40 C^{2}+2.64 A B-1.50 A C-0.71 A D-3.54 B C-0.59 B D+0.81 C D
\end{aligned}
$$

$$
Y_{2}=3.68+0.053-0.54 B+9.175 E-0.03 C-0.45 A B-0.19 A C-0.69 B C
$$

Positive values in these equations reflect an enhancement of the response by the experimental variable, while a negative value means the experimental variable decreased the response.

As shown in Figure 2, the size distribution of the pure standard piroxicam powder was broad, while the size distributions of the microcrystals of the optimized formulations were narrow and uniform. The same results were obtained in other studies for gliclazide (27), ibuprofen (29), itraconazole and ketoconazol (11), and indomethacine (16). The narrow and uniform size distribution of the particles is one of the important advantages of the microcrystallization process, in contrast with other particle size reduction methods such as milling (16). Statistical analysis of the particle size of microcrystals produced by the solvent-change method by a two-way ANOVA test shows that the drug and stabilizing agent concentrations and the type of stabilizer had a significant effect $(p<0.05)$ on particle size, while the homogenization speed did not have a significant effect on particle size $(p>0.05)$.

The statistical analysis by a two-way ANOVA shows that increasing the drug concentration from $0.03 \%$ to $0.45 \%$ or the stabilizer concentration from $0.04 \%$ to $0.06 \%$ had a lowering effect on particle size, while changing the Brij35 to poloxamer, increasing the concentration of the drug from $0.45 \%$ to $0.6 \%$, or increasing the stabilizer concentration form $0.03 \%$ to $0.04 \%$ had an additive effect on the particle size of the microcrystals prepared by the solventchange method.

The two-way statistical analysis of particle size for microcrystals produced by the $\mathrm{pH}$-shift method shows that drug concentration and homogenization speed had insignificant effects on particle size $(p>0.05)$, while the stabilizing agent concentration had a significant effect on this variable $(p<0.05)$. The analysis of variance shows that increasing the stabilizing agent concentration had a lowering effect on the particle size.

\section{Dissolution Studies}

Dissolution profiles of untreated piroxicam and crystals obtained from the two microcrystallization methods were obtained in $\mathrm{pH} 1.2$ medium (Figure 3 ). The $\mathrm{DE}_{150}$ was calculated from the dissolution data as an independent release model parameter (Tables 2 and 3). All treated samples had remarkably faster dissolution rates than the untreated drug. The highest $\mathrm{DE}_{150}$ value in the solventchange method was from the formulation $\mathrm{P}_{0.3} \mathrm{P}_{0.06} \mathrm{~S}_{21500 \text {, }}$ while formulation $\mathrm{P}_{0.3} \mathrm{~B}_{0.06} \mathrm{~S}_{17500}$ had the highest value in the $\mathrm{pH}$-shift method (Tables 2 and 3 ).

A linear model to predict the $D E_{150}$ value $\left(Y_{3}\right.$ and $\left.Y_{4}\right)$ of the microcrystals was suggested by the Design Expert software. According to this model, the final equations are as follows.

For the solvent-change method:

$$
Y_{3}=66.89-5.55 A+1.10 B+9.80 C+0.41 D
$$

For the $\mathrm{pH}$-shift method:

$$
\mathrm{Y}_{4}=67.17-2.32 A+0.52 B-3.74 C
$$

Dissolution rates of all formulations prepared by the solvent-change and $\mathrm{pH}$-shift methods were faster than those of the untreated powder (Tables 2 and 3). Particles size, crystalline form, and the $\mathrm{pH}$ of the dissolution medium are considered the main determinant factors for dissolution rate (16). In all microcrystals of piroxicam, higher

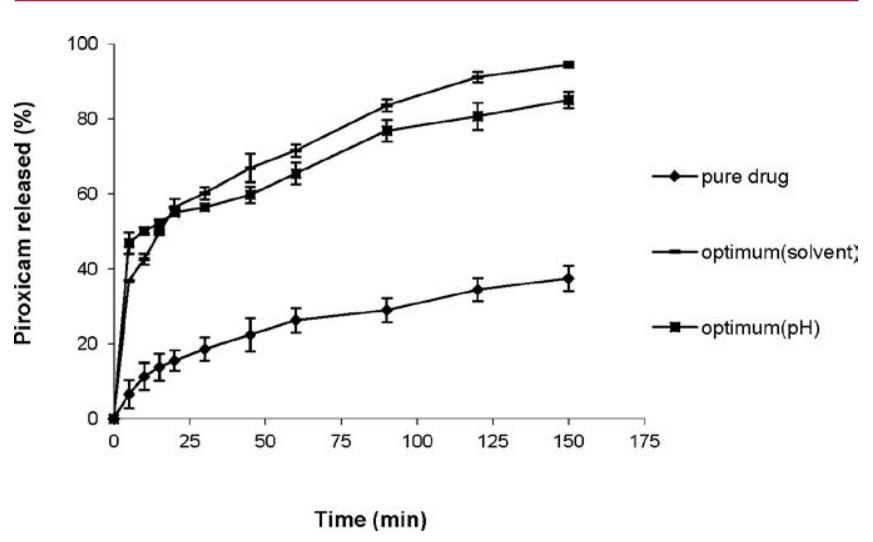

Figure 3. Drug dissolution profiles of untreated piroxicam powder compared with its optimized microcrystals prepared by solvent-change and pH-shift methods in $\mathrm{pH} 1.2(n=3)$. 

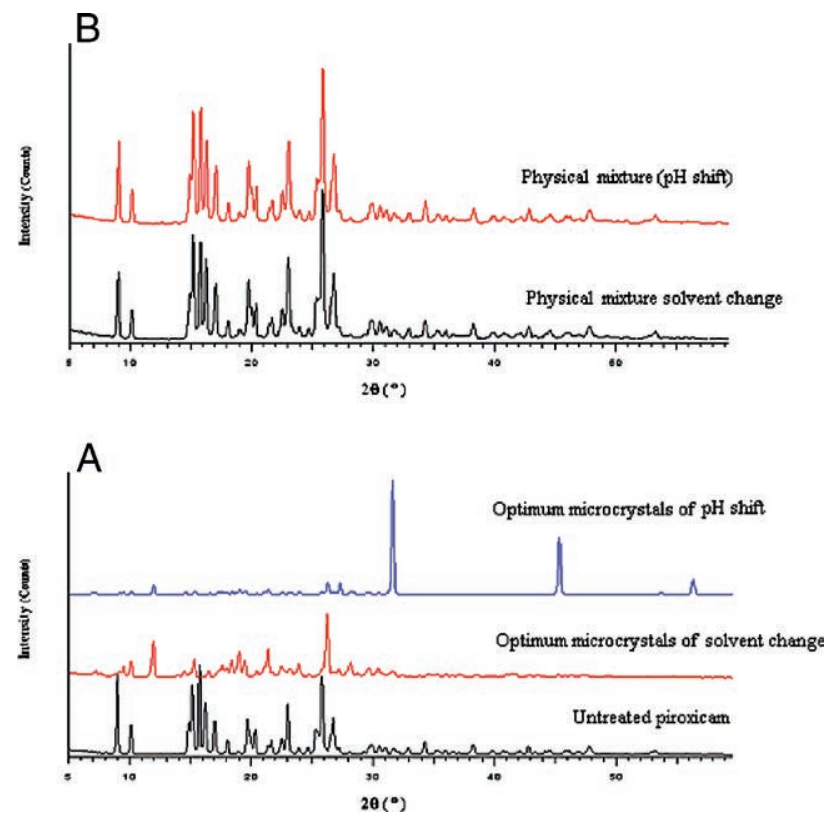

Figure 4. $X$-ray diffraction patterns of $(A)$ untreated piroxicam powder and optimized microcrystals of piroxicam prepared by solvent-change and $\mathrm{pH}$ shift methods and $(B)$ the physical mixture of piroxicam with additives of each method.

dissolution rates appeared to result from reduced particle size (Tables 2 and 3 ) and a subsequently increased surface area, as shown in Table 4 (24).

In the solvent-change method, $\mathrm{DE}_{150}$ decreased with an increase in drug concentration $(p<0.05)$ and increased with an increase in homogenization speed $(p<0.05)$. However, the effects of the stabilizing agent concentration and the type of polymer were not statistically significant $(p>0.05)$, as shown in Table 2 .

The drug and stabilizing agent concentration, homogenization speed, and stabilizer type had a significant effect $(p<0.05)$ on $\mathrm{DE}_{150}$ of gliclazide microcrystals prepared by the solvent-change method (30). A linear model was fitted to the effect of drug concentration and homogenization speed on $\mathrm{DE}_{150}$. Accordingly, $\mathrm{DE}_{150}$ decreased linearly with increasing drug concentration at all homogenization speeds. Mixing the drug powder with polymers resulted in increased wettability and dissolution surface area and reduced interfacial tension between the dissolution medium and the hydrophobic drug surface (30).

\section{Optimized Formulations}

Computer optimization of the results from response surface methodology by D-optimal design (Tables 2 and 3) will allow the estimation of a specific combination of variables $\left(X_{i}\right)$ that will optimize the individual responses $\left(Y_{i}\right)$ and will yield a product with desirable qualities. The variables involved in the optimization of all studied factors were particle size and $D_{150}$ of the microcrystals. The optimal values (i.e., 100\% desirability) were predicted by Design Expert software for particle size $\left(\mathrm{Y}_{1}, \mathrm{Y}_{2}\right)$ and $\mathrm{DE}_{150}\left(\mathrm{Y}_{3}\right.$, $\mathrm{Y}_{4}$ ) for the solvent-change and $\mathrm{pH}$-shift methods, respectively. To achieve these levels, the predicted formulations of microcrystals were $\mathrm{P}_{0.6} \mathrm{P}_{0.06} \mathrm{~S}_{21500}$ (for solvent change) and $\mathrm{P}_{0.3} \mathrm{~B}_{0.03} \mathrm{~S}_{17500}$ (for $\mathrm{pH}$-shift method). The particle size and $D_{150}$ of the optimized microcrystals are shown in Table 4. Statistical analysis of the results by independent Student's $t$-test shows that the particle size obtained by the solvent-change method is significantly smaller than that obtained by the $\mathrm{pH}$-shift method $(p<0.05)$. $\mathrm{DE}_{150}$ is also significantly greater for the solvent-change method than for the $\mathrm{pH}$-shift method $(p<0.05)$. Consequently, the solvent-change method is preferred for the production of piroxicam microcrystals.

\section{X-ray Diffraction Studies}

Diffraction spectra of untreated piroxicam powder, optimized microcrystal formulations from each preparation method, and their physical mixtures are shown in Figure 4. The XRD patterns of the physical mixtures are very similar to that of the standard crystalline powder. The major peaks are present in the diffractograms of the optimized formulations but have less intensity than those for the untreated crystalline drug. Figure 3 shows that the XRD patterns of the physical mixtures of the optimized formulations and the untreated drug powder are completely similar. Because peak height is influenced by crystal size and crystallinity, the reduction of the height of the peaks indicates reduction of the particle size and formation of the microcrystalline form of the drug (31). Gliclazide microcrystals also showed such a phenomenon in their XRD patterns (27).

$\mathrm{NaCl}$ crystals could be responsible for sharp peaks in $2 \theta=30$ (Figure 4) from microcrystals produced by the $\mathrm{pH}$ shift method, as $\mathrm{HCl}$ and $\mathrm{NaOH}$ were used to adjust the $\mathrm{pH}$. Treated phenytoin powder showed more peaks in the XRD pattern than the untreated drug powder (32). This could be due to an alteration of the powder to a completely

Table 4. Results of Thermal Analysis and Surface Area of Optimized Piroxicam Microcrystals Prepared by Solvent-Change and pH-Shift Methods

\begin{tabular}{|c|c|c|c|c|c|c|}
\hline Formulation Code & $\begin{array}{l}\text { Particle size } \\
(\text { nm) }\end{array}$ & $\begin{array}{l}\mathrm{DE}_{150} \\
(\%)\end{array}$ & $\begin{array}{c}T_{\mathrm{m}} \\
\left({ }^{\circ} \mathrm{C}\right)\end{array}$ & $\begin{array}{c}\Delta H \\
(\mathbf{j} / \mathbf{g})\end{array}$ & $\begin{array}{c}\text { Surface area } \\
\left(\mathrm{m}^{2} / \mathrm{g}\right)\end{array}$ & $\begin{array}{c}V_{m} \\
(\mathrm{~mL} / \mathrm{g})\end{array}$ \\
\hline Untreated piroxicam & $15.6 \pm 37.4$ & $24.0 \pm 4.4$ & 199.4 & 685.5 & 1.98 & 0.0005 \\
\hline Optimized microcrystals of solvent-change method $\left(\mathrm{P}_{0.6} \mathrm{P}_{0.06} \mathrm{~S}_{21500}\right)$ & $2.17 \pm 0.3$ & $81.0 \pm 5.6$ & 197.4 & 630.89 & 2.10 & 0.0006 \\
\hline Optimized microcrystals of $\mathrm{pH}$-shift method $\left(\mathrm{P}_{0.3} \mathrm{~B}_{0.03} \mathrm{~S}_{17500}\right)$ & $3.75 \pm 0.46$ & $69.0 \pm 2.9$ & 190.5 & 902.58 & 4.39 & 0.001 \\
\hline
\end{tabular}



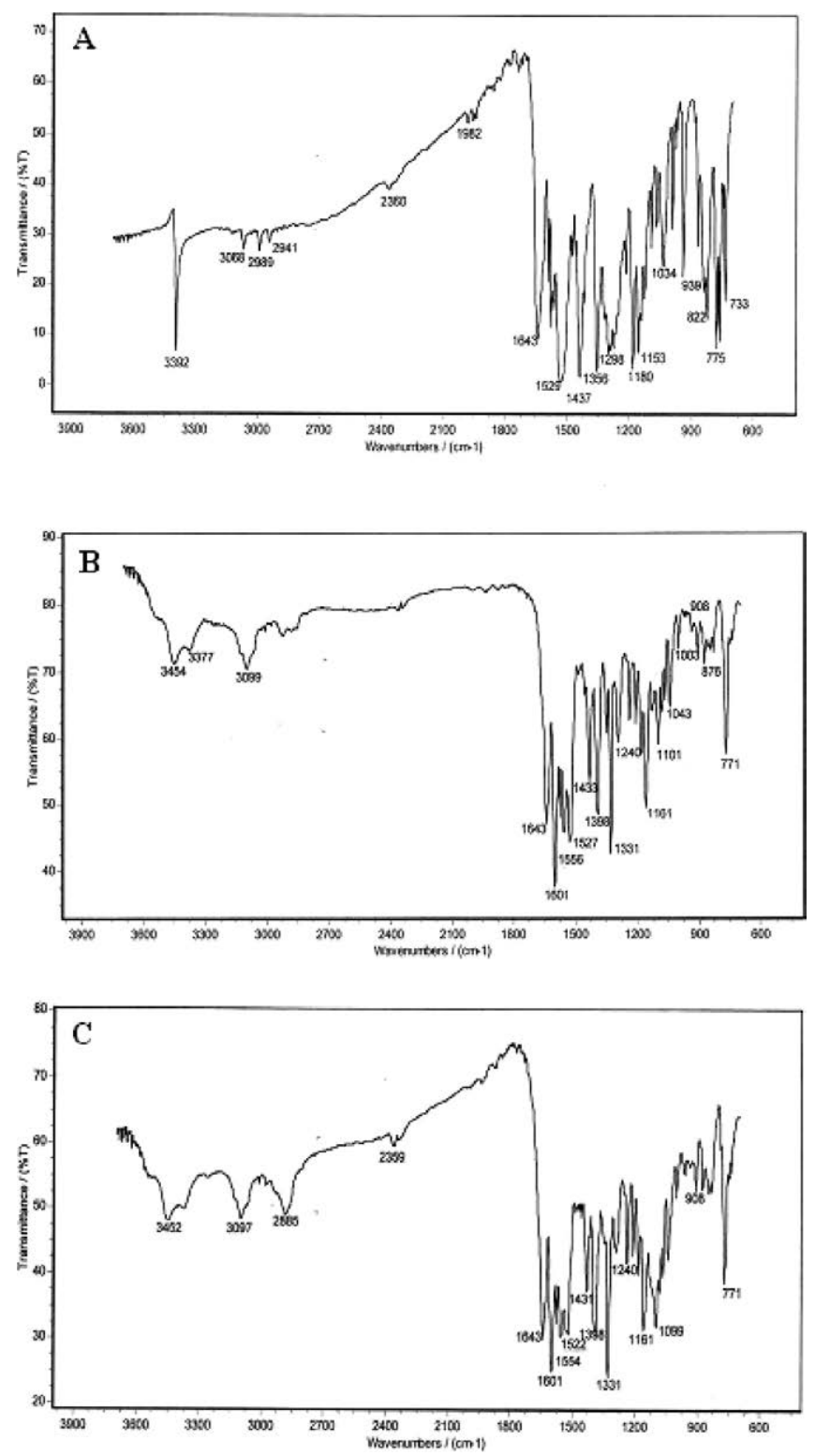

Figure 5. FTIR spectra of (A) untreated piroxicam powder, (B) optimized microcrystals of piroxicam prepared by solvent-change method, and (C) optimized microcrystals of piroxicam prepared by $\mathrm{pH}$-shift method.

crystalline form or a difference in crystal orientation due to a change in crystalline habit. However, a lack of difference between the XRD patterns might suggest that there is no change in the drug crystallinity and no interaction between the drug and excipients during the procedure. This could also be understood by studying the DSC thermograms.

\section{FTIR Spectroscopy}

FTIR spectroscopy was used characterize possible changes in the chemical structure and drug-additive interactions in the untreated crystalline powder and the microcrystals. The spectra of all samples are similar (Figure 5). The principle absorption bands of piroxicam appear in
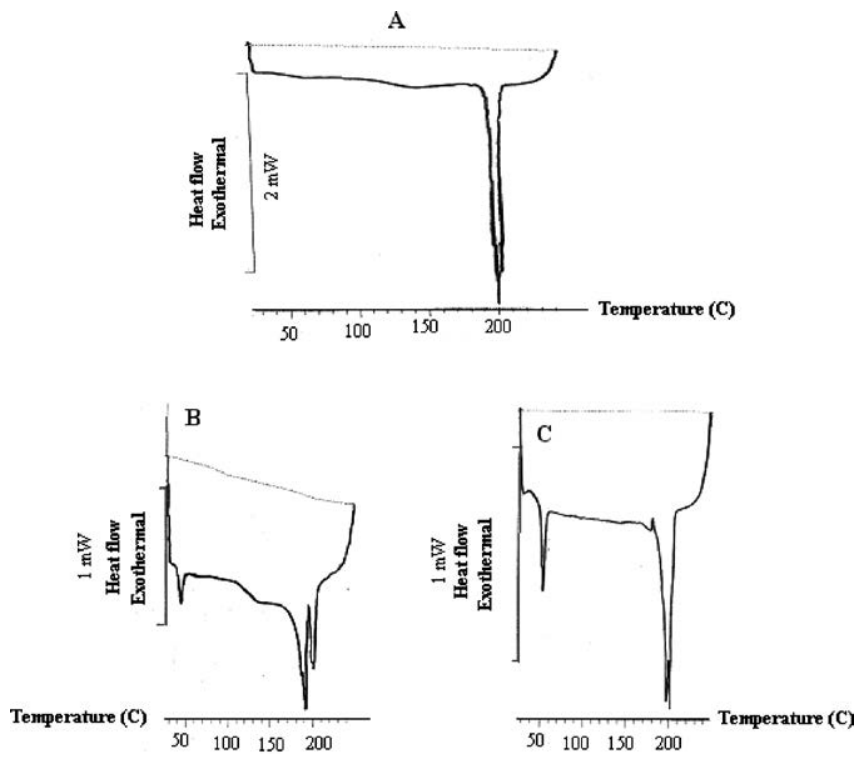

Figure 6. DSC thermograms of (A) untreated piroxicam powder, (B) optimized microcrystals of piroxicam prepared by solvent-change method, and (C) optimized microcrystals of piroxicam prepared by $\mathrm{pH}$-shift method.

the regions of 3392,1643 , and $1356 \mathrm{~cm}^{-1}$, and these bands are related to the functional groups of $\mathrm{OH}, \mathrm{C}=\mathrm{O}$, and $\mathrm{S}=\mathrm{O}$, respectively (Figure $5 \mathrm{~A}$ ).

These bands were slightly shifted to the regions of 3354, 1643 , and $1331 \mathrm{~cm}^{-1}$ in the FTIR spectra of microcrystals prepared by the solvent-change method (Figure 5B) and to the regions of 3452,1643 , and $1331 \mathrm{~cm}^{-1}$ for the $\mathrm{pH}-$ shift method (Figure 5C). As there is no significant difference in the location of the bands, the internal structures were not different (27), and there was no incompatibility between the drug and stabilizers (33).

\section{DSC Studies}

Figure 6 shows the DSC thermograms of untreated piroxicam and its optimum microcrystals. The final results for melting point and enthalpy are also listed in Table 4. The $T_{\mathrm{m}}$ values for the drug are similar in all experiments and consistent with the literature (34). The presence of similar endothermic peaks, which are associated with the melting points, suggests no interaction between the drug and excipients and no change in drug crystallinity (20). The endothermic peak at $200^{\circ} \mathrm{C}$ relates to the melting point of the drug (Figure $6 \mathrm{~A}$ ). The $T_{\mathrm{m}}$ peak shows only a slight but insignificant change. If the main peaks were omitted, this could mean that the entire product was changed to the amorphous form (35). A slight reduction in $T_{\mathrm{m}}$ indicates that microcrystals are not completely crystalline (16). In addition, a change in the melting point may suggest the presence of polymers $(17,36,37)$. Short peaks at the beginning of the heating procedure (Figure $6 \mathrm{~B}, \mathrm{C}$ ) could be related to poloxamer and Brij melting points (28, 38,39 ). 
The third endothermic peak in Figure 6A might suggest the presence of small amounts of drug polymorphic form. This was also observed for the solid dispersion of carbamazepine (35).

Table 4 shows a reduction in enthalpy changes of optimized microcrystals from the solvent-change method relative to the untreated drug. The reduction in microcrystal particle size and the small amount of stabilizer might affect a reduction in enthalpy (27). Perhaps the precipitated drug is sterically stabilized against crystal growth by adsorbed polymers (Brij or Poloxamer), and the surface energy and consequently the enthalpy of the system is lowered. Accordingly, the molecularly dispersed drug is associated with particles in the required size range and simultaneously stabilized in the formed dispersion. Chow et al. (40) also have reported that the modification of the physical properties of phenytoin by recrystallization from methanol exhibited an increase in the specific surface area of the phenytoin crystals, a drastic reduction in crystallization yield, and a progressive change of the crystal habit from needles to elongated plates. Powder X-ray diffraction studies on the samples indicated essentially the same diffraction patterns and lattice spacing for both the untreated phenytoin powder and its recrystallized form, suggesting that the doped crystals did not undergo gross structural modification. However, the enthalpy of fusion, $H_{\mathrm{f}}$, as determined by DSC, was reduced by as much as $17 \%$, indicating a significant change in both the enthalpy and entropy of the phenytoin crystals.

Enthalpy changes of the optimized formulation of microcrystals from the $\mathrm{pH}$-shift method were higher than those of the untreated drug (Table 4). The presence of dissolved impurities may affect the rate of crystallization and even change the crystal habit, provided that these impurities are surface active and become adsorbed onto the nuclei or onto growing crystals (41). The microcrystals from the $\mathrm{pH}$-shift method had a higher enthalpy than the untreated drug, which might result from the presence of impurities of the $\mathrm{NaCl}$ produced.

\section{Specific Surface Area}

According to the BET equation (25), the specific surface area and the monolayer adsorbed gas volume were calculated for the untreated piroxicam powder and the two optimized formulations of each preparation method (Table 4). The results show an increase in the surface area of the microcrystals in comparison with the untreated drug powder that is consistent with the increase in the specific surface area and the monolayer adsorbed gas volume (Table 4).

\section{CONCLUSION}

The results of the present study show that microcrystallization has an effect on piroxicam crystal habit modification. Both the $\mathrm{pH}$-shift and the solvent-change methods using an ultra-homogenizer and stabilizers produced microcrystals with a higher dissolution rate than those prepared with pure piroxicam powder. The highest dissolution rate and the lowest particle size were observed using the solvent-change method.

\section{ACKNOWLEDGMENTS}

The authors wish to thank the Vice Chancellery of Isfahan University of Medical Sciences that supported this work by project No. 386253.

\section{REFERENCES}

1. Gursoy, R. N.; Benita, S. Self-emulsifying drug delivery systems (SEDDS) for improved oral delivery of lipophilic drugs. Biomed. Pharmacother. 2004, 58 (3), 173-182.

2. Lipinski, C. A. L. F.; Dominy, B. W.; Feeney, P. J. Poor aqueous solubility: An industry-wide problem in drug discovery. Am. Pharm. Rev. 2002, 5 (1), 82-85.

3. Löbenberg, R.; Amidon, G. L. Modern bioavailability, bioequivalence and biopharmaceutics classification system. New scientific approaches to international regulatory standards. Eur. J. Pharm. Biopharm. 2000, 50 (1), 3-12.

4. Orienti, I.; Bigucci, F.; Luppi, B.; Cerchiara, T.; Zuccari, G.; Giunchedi, P.; Zecchi, V. Polyvinylalcohol substituted with triethyleneglycolmonoethylether as a new material for preparation of solid dispersions of hydrophobic drugs. Eur. J. Pharm. Biopharm. 2002, 54 (2), 229-233.

5. Javadzadeh, Y.; Siahi, M. R.; Asnaashari, S.; Nokhodchi, A. An investigation of physicochemical properties of piroxicam liquisolid compacts. Pharm. Dev. Technol. 2007, 12 (3), 337-343.

6. Cavallari, C.; Abertini, B.; Gonzalez, M.; Rodriguez, L. Improved dissolution behaviour of steam-granulated piroxicam. Eur. J. Pharm. Biopharm. 2002, 54 (1), 65-73.

7. Javadzadeh, Y.; Siahi-Shadbad, M. R.; Barzegar-Jalali, M.; Nokhodchi, A. Enhancement of dissolution rate of piroxicam using liquisolid compacts. Farmaco 2005, 60 (4), 361-365.

8. Albertini, B.; Cavallari, C.; Passerini, N.; GonzalezRodriguez, M. L.; Rodriguez, L. Evaluation of betalactose, PVP K12 and PVP K90 as excipients to prepare piroxicam granules using two wet granulation techniques. Eur. J. Pharm. Biopharm. 2003, 56 (3), 479-487.

9. Verma, M. M.; Kumar, M. T.; Balasubramaniam, J.; Pandit, J. K. Dissolution, bioavailability and ulcerogenic studies on piroxicam-nicotinamide solid dispersion formulations. Boll. Chim. Farm. 2003, 142 (3), 119-124.

10. Pan, R. N.; Chen, J. H.; Chen, R. R. Enhancement of dissolution and bioavailability of piroxicam in solid dispersion systems. Drug Dev. Ind. Pharm. 2000, 26 (9), 989-994.

11. Rasenack, N.; Muller, B. W. Dissolution rate enhancement by in situ micronization of poorly water-soluble drugs. Pharm. Res. 2002, 19 (12), 1894-1900.

12. Rogers, T. L.; Overhoff, K. A.; Shah, P.; Santiago, P.; Yacaman, M. J.; Johnston, K. P.; Williams, R. O. 
Micronized powders of a poorly water soluble drug produced by a spray-freezing into liquid-emulsion process. Eur. J. Pharm. Biopharm. 2003, 55 (2), 161-172.

13. Finholt, P.; Solvang, S. Dissolution kinetics of drugs in human gastric juice-the role of surface tension. J. Pharm. Sci. 1968, 57 (8), 1322-1326.

14. Nobuyoshi, K.; Akiko, I. Influence of Ball-Milling Atmosphere on Decrease of Molecular Weight of Polyvinylpyrrolidone Powders. Chem. Pharm. Bull. 1972, 20 (7), 1536-1543.

15. Waltersson, J. O.; Lundgren, P. The effect of mechanical comminution on drug stability. Acta Pharm. Suec. 1985, 22 (5), 291-300.

16. Kim, S. T.; Kwon, J. H.; Lee, J. J.; Kim, C. W. Microcrystallization of indomethacin using a pH-shift method. Int. J. Pharm. 2003, 263 (1-2), 141-150.

17. Rasenack, N.; Hartenhauer, H.; Müller, B. W. Microcrystals for dissolution rate enhancement of poorly water-soluble drugs. Int. J. Pharm. 2003, 254 (2), 137-145.

18. Palakodaty, S.; York, P. Phase behavioral effects on particle formation processes using supercritical fluids. Pharm. Res. 1999, 16 (7), 976-985.

19. York, P. Strategies for particle design using supercritical fluid technologies. Pharm. Sci. Technol. Today 1999, 2 (11), 430-440.

20. Javadzadeh, Y.; Jafari-Navimipour, B.; Nokhodchi, A. Liquisolid technique for dissolution rate enhancement of a high dose water-insoluble drug (carbamazepine). Int. J. Pharm. 2007, 341 (1-2), 26-34.

21. Rasenack, N.; Steckel, H.; Müller, B. W. Preparation of microcrystals by in situ micronization. Powder Technol. 2004, 143, 291-296.

22. Steckel, H.; Rasenack, N.; Müller, B. W. In-situmicronization of disodium cromoglycate for pulmonary delivery. Eur. J. Pharm. Biopharm. 2003, 55 (2), 173-180.

23. Zimmermann, A.; Millqvist-Fureby, A.; Elema, M. R.; Hansen, T.; Mullertz, A.; Hovgaard, L. Adsorption of pharmaceutical excipients onto microcrystals of siramesine hydrochloride: effects on physicochemical properties. Eur. J. Pharm. Biopharm. 2009, 71 (1), 109-116.

24. Affonso, A.; Naik, V. R. Microcrystallization methods for aspirin, mebutamate, and quinine sulfate. J. Pharm. Sci. 1971, 60 (10), 1572-1574.

25. Brunauer, S. E. P.; Teller, E. BET theory. J. Am. Chem. Soc. 1938, 60, 309-319.

26. Costa, P.; Manuel, J.; Lobo, S. Modeling and comparison of dissolution profiles. Eur. J. Pharm. Sci. 2001, 13, 123-133.

27. Varshosaz, J.; Talari, R.; Mostafavi, S. A.; Nokhodchi, A. Dissolution enhancement of gliclazide using in situ micronization by solvent change method. Powder Technol. 2008, 187 (3), 222-230.
28. Rowe, R. C.; Sheskey, P. J.; Weller, P. J.; Rowe, R. Handbook of Pharmaceutical Excipients, 4th ed.; APhA Publications: Chicago, 2003.

29. Rasenack, N.; Müller, B. W. Ibuprofen crystals with optimized properties. Int. J. Pharm. 2002, 245 (1-2), 9-24.

30. Yagi, N.; Terashima, Y.; Kenmotsu, H.; Sekikawa, H.; Takada, M. Dissolution Behavior of Probucol from Solid Dispersion Systems of Probucol-Polyvinylpyrrolidone. Chem. Pharm. Bull. 1996, 44 (1), 241-244.

31. Pharmaceutical Preformulation and Formulation: A Practical Guide from Candidate Drug Selection to Commercial Dosage Form; Gibson, M., Ed.; Informa Healthcare: London, 2001.

32. Nokhodchi, A.; Bolourtchian, N.; Dinarvand, R. Crystal modification of phenytoin using different solvents and crystallization conditions. Int. J. Pharm. 2003, 250 (1), 85-97.

33. Jovanovic, N.; Bouchard, A.; Hofland, G. W.; Witkamp, G. J.; Crommelin, D. J.; Jiskoot, W. Stabilization of proteins in dry powder formulations using supercritical fluid technology. Pharm. Res. 2004, 21 (11), 1955-1969.

34. Clarke's Analysis of Drugs and Poisons, 3rd ed.; Moffat, A. C., Osselton, M. D., Widdop, B., Eds.; Pharmaceutical Press: London, 2004.

35. Rane, Y.; Mashru, R.; Sankalia, M.; Sankalia, J. Effect of hydrophilic swellable polymers on dissolution enhancement of carbamazepine solid dispersions studied using response surface methodology. AAPS PharmSciTech 2007, 8 (2), E1-E11.

36. Chiou, W. L.; Riegelman, S. Pharmaceutical applications of solid dispersion systems. J. Pharm. Sci. 1971, 60 (9), 1281-1302.

37. Papageorgiou, G. Z.; Bikiaris, D.; Karavas, E.; Politis, S.; Docoslis, A.; Park, Y.; Stergiou, A.; Georgarakis, E. Effect of physical state and particle size distribution on dissolution enhancement of nimodipine/PEG solid dispersions prepared by melt mixing and solvent evaporation. AAPS J. 2006, 8 (4), E623-E631.

38. Hillgren, A.; Alden, M. A comparison between the protection of LDH during freeze-thawing by PEG 6000 and Brij 35 at low concentrations. Int. J. Pharm. 2002, 244 (1-2), 137-149.

39. Passerini, N.; Albertini, B.; Gonzalez-Rodriguez, M. L.; Cavallari, C.; Rodriguez, L. Preparation and characterisation of ibuprofen-poloxamer 188 granules obtained by melt granulation. Eur. J. Pharm. Sci. 2002, 15 (1), 71-78.

40. Chow, A. H. L.; Gordon, J. D.; Szeitz, A.; Young, J. W. M. Modification of phenytoin crystals. III. Influence of 3-butanoyloxymethyl-5,5-diphenylhydantoin on solution-phase crystallization and related crystal properties. Int. J. Pharm. 1995, 126 (1-2), 11-19.

41. Bowman, B. J.; Ofner, C. M.; Schott, H. Colloidal Dispersions. In Remington: The Science and Practice of Pharmacy, 21st ed.; Troy, D. B., Ed.; Lippincott Williams \& Wilkins: Philadelphia, 2005; p 307. 